\title{
Rejoinder on: Comparing and selecting spatial predictors using local criteria
}

\author{
Jonathan R. Bradley • Noel Cressie · Tao Shi
}

Published online: 29 November 2014

(C) Sociedad de Estadística e Investigación Operativa 2014

\section{Introduction}

First and foremost, we would like to thank the discussants: Stefano Castruccio and Marc G. Genton (CG), Alan E. Gelfand (GE), Finn Lindgren (LI), M.D. Ruiz-Medina (RM), and Martin P. Tingley and Ben A. Shaby (TS) for giving generously of their time to comment on our paper, "Comparing and Selecting Spatial Predictors Using Local Criteria" by Jonathan R. Bradley, Noel Cressie, and Tao Shi (BCS). Their comments and those of the editors and referees have given us this opportunity to amplify and clarify some aspects of using local criteria to compare and select spatial predictors.

In BCS, we propose local criteria to compare and select from a finite number of spatial predictors defined on a (possibly irregular) spatial lattice $D \equiv\left\{\mathbf{u}_{1}, \ldots, \mathbf{u}_{N}\right\} \subset$ $\mathbb{R}^{d}$. These criteria lead naturally to the idea of selecting predictors optimally, locationby-location; the result is a hybrid spatial predictor, which we call a locally selected predictor (LSP), that has the potential to perform better than any individual predictor.

This rejoinder refers to the comments available at doi:10.1007/s11749-014-0385-3; doi:10.1007/s11749-014-0411-5; doi:10.1007/s11749-014-0412-4; doi:10.1007/s11749-014-0413-3; doi:10.1007/s11749-014-0417-z.

\section{J. R. Bradley $(\varangle)$}

Department of Statistics, University of Missouri, 146 Middlebush Hall, Columbia, MO 65211, USA

e-mail: bradleyjr@missouri.edu

\section{N. Cressie}

National Institute for Applied Statistics Research Australia,

University of Wollongong, Wollongong, Australia

T. Shi

Department of Statistics, The Ohio State University, Columbus, OH, USA 
Furthermore, this local selection allows us to see at which locations an individual spatial predictor is performing comparatively well.

Notice that the LSP serves two main roles, namely, spatial prediction and "local" comparison of different (global) spatial predictors. Hence, identifying regions where a predictor performs well gives us another way to assess non-stationarity in the underlying physical process. Thus, we agree with CG and TS that local comparisons add to the understanding of the physical process; see Sections 1, 3.2, and 6 of BCS for a discussion on using $\hat{k}(\cdot)$ for this purpose.

A strength of our approach is that it allows one to compare and select predictions derived from very different assumptions. GE acknowledges these advantages but expresses a preference for full stochastic modeling. While we appreciate GE's point of view, this era of big, spatial, non-stationary data opens up other exciting possibilities; see Section 1 of BCS.

With both training data and validation data at hand, we can consider any candidate predictor, be it deterministic or stochastic, to improve spatial predictions and our understanding of the spatial variability in the underlying physical process. This algorithmic point of view increases the utility of the LSP, but it can be difficult to conceptualize in the abstract (as GE mentions). However, it is needed since it is common for multiple researchers to be motivated by different (and worthwhile) perspectives, leading them to propose different spatial predictors (often arising from different stochastic models). For example, we were primarily interested in the predictors introduced in Section 3.1 of BCS. However, TS suggest using predictors estimated locally, proposing that this aids computation and possibly interpretation; our approach is flexible enough to allow for these predictors (if they are of interest). Certainly, considering local features of global statistics is a rational approach that has quite a long history in the analysis of spatial data (e.g., Anselin 1995).

Both CG and LI were interested in seeing the heterogeneous version of SPD as opposed to the homogeneous version used in BCS. Again, our methodology is flexible enough to include the former as a candidate predictor. A map of the heterogeneous SPD predictions is given in Fig. 1, which has RSTE equal to 4.1700. The RSTE for

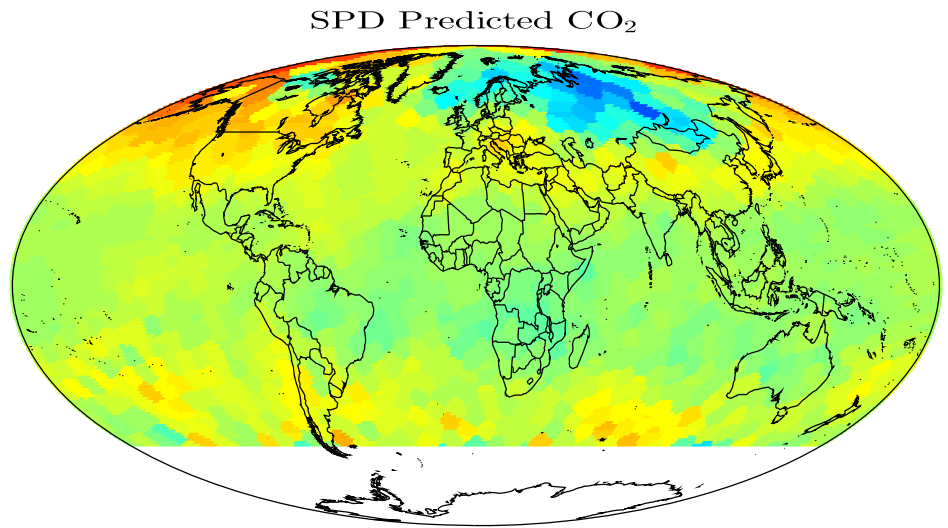

Fig. 1 Global spatial prediction of mid-tropospheric $\mathrm{CO}_{2}$ concentrations using the heterogeneous version of SPD. Note that we do not predict below latitude $-60^{\circ}$, since AIRS has not released any observations there 
LSP-MWS (using the heterogeneous SPD in place of its homogeneous version) is 4.0018; that is, when compared to 3.9652 for the homogeneous version given in Table 3 of BCS, we see a slight increase in RSTE.

The candidate set of predictors is one general theme of the discussants' comments. Other themes include the role of big data, alternative LSP specifications, and uncertainty quantification. We add our comments to these themes in Sections 2 through 5 below, and we provide concluding remarks in Section 6.

\section{The candidate set of predictors}

We have pointed out in this rejoinder that any predictor for which an algorithm exists to compute $\hat{Y}^{\mathrm{PRD}}\left(\mathbf{s}_{0}\right)$, for all $\mathbf{s}_{0} \in D$, qualifies as a candidate predictor. Some are motivated by smoothing, such as one might find in GIS packages, and some are motivated by optimality under assumptions about the hidden spatial process (e.g., kriging).

Two of the discussants (CG and TS) raised doubts about one of the predictors included, namely FRK, referencing Stein (2014): Our point above is that it qualifies algorithmically. Now, the FRK predictor due to Cressie and Johannesson (2006, 2008) is optimal under a spatial random effects (SRE) model, but Stein (2014) has written an essay that concentrates on the likelihood and estimation of parameters under the SRE model. Consequently, he considers only properties of the marginal distribution $[\mathbf{Z} \mid \boldsymbol{\theta}]$ and not of the predictive distribution $\left[Y\left(\mathbf{s}_{0}\right) \mid \mathbf{Z}, \boldsymbol{\theta}\right]$, where $\boldsymbol{\theta}$ are the SRE-model parameters. Here we bring the reader's attention to Zimmerman and Zimmerman (1991), where the difference between estimation and prediction in spatial statistics is carefully discussed. The small amount of prediction discussed in Stein's paper considers the scientifically less-interesting problem of prediction of $Z\left(\mathbf{s}_{0}\right)$ given $\mathbf{Z}$ and $\boldsymbol{\theta}$; see Cressie and Wikle (2011, p. 42) for a more complete discussion of why it is less interesting. Further, when comparing estimation of $\boldsymbol{\theta}$ from different models, Stein fails to adjust the likelihood to account for the number of parameters, such as is done through AIC.

The SRE model is highly flexible, allowing Stein to choose almost-degenerate special cases. Specifically, he uses mathematical and simulation results for periodic processes in one-dimensional space with strong assumptions about large- $n$ behavior; he uses only smooth orthogonal basis functions, but they could in fact be nonorthogonal and non-continuous; he assumes situations where the SRE model should not be used, such as near-zero measurement error; and so forth. Every stochastic model has its limitations, but they do not always affect the efficiency of its associated predictor and, indeed, we see from the simulation experiment and the real example in BCS that FRK is a highly competitive predictor among the seven chosen.

\section{The role of big data}

We agree with GE that our method is most appropriate for the setting where the sample size is greater than 1,000. In Section 1 of BCS, we motivate the LSP through the lens 
of spatial prediction using big data; see the additional stimulating comments given by CG. While we agree with GE that prediction is not the only type of statistical inference that is important for big spatial data, this is what we do in BCS.

The stochastic models, used to derive computable spatial predictors for big spatial data, are motivated by the idea of striking a balance between preserving data structure and reducing the complexity of the model. For example, covariance tapering ignores long-distance correlation and regularizes the covariance matrix, separable covariances ignore space-time interactions, composite-likelihood methods partially order the spatial domain to ignore spatial dependence between observations far apart, full-rank methods reduce the parameter space, and reduced-rank methods use a reduced set of spatial frequencies [see Sun et al. (2012), for a more in-depth discussion]. These modeling compromises are generally made so that statistical inference is practical on big spatial data.

From this perspective, the LSP is especially valuable. While each individual spatial predictor might ignore certain "types of valuable information," the hybrid LSP uses the totality of information from all predictors with very little extra computing required. Hence, the candidate predictors that define the LSP might be chosen to complement each other in terms of their strengths and limitations. This adds motivation for considering both reduced-rank and full-rank methods in Section 5 of BCS.

\section{Alternative LSP specifications}

\section{$4.1 D^{\mathrm{val}}$}

GE expressed reservations about the specification of training and validation datasets. The appropriateness of whether or not to separate data into training, validation, and testing is largely dependent on the amount of data available (e.g., see Hastie et al. 2009, p. 222). The data used in BCS can be separated in this way since we are in a big data setting.

In response to GE's question, we are interested in addressing the problem of overfitting, which is precisely the reason why one would separate data into training, validation, and testing (e.g., see Hastie et al. 2009, Ch. 7, on how the separation of data relates to over-fitting). Also, creation of a testing dataset is not unconventional, and it is covered in standard textbooks (e.g., see Hastie et al. 2009, p. 222).

The appropriate specification of training, validation, and testing data is an open problem in our LSP approach; see Section 4.2 of BCS, where we incorporate it into the simulation experiment. An interesting suggestion was given by RM: Specifically, a "bagging" approach (Hastie et al. 2009) could be used to consider multiple training and validation datasets. That is,

1. Randomly generate $D^{\text {trn }}$ and $D^{\text {val }}$ from within $D$;

2. Compute the LSP;

3. Repeat Steps 1. and 2. B times to create a distribution of LSPs;

4. Average across the $B$ LSPs generated in Step 3. 
We see promise in this idea but note that it may be difficult to get $B$ right. Re-computing each individual spatial predictor $B$ times may be computationally difficult in many settings. As a result, this might restrict one to using small values for $B$.

\section{$4.2 H^{\mathrm{val}}$}

LI comments that $H^{\mathrm{val}}$ is not interpretable when the spatial domain $D$ is defined in continuous space; he uses the terminology "punctured" to describe the definitions of $H^{\mathrm{val}}$ in Section 2.3 of BCS. However, it is important to note that $D$ is defined as $N$ discrete spatial locations in $\mathbb{R}^{d}$ (see Section 2.1 of BCS). Furthermore, the twostage definitions of $H^{\mathrm{val}}$ in Section 2.3 of BCS leads to an optimality result (see the Appendix of BCS), which may not hold for LI's suggested version of $H^{\mathrm{val}}$.

\subsection{The selection criterion}

We are excited and encouraged by LI's consideration of alternative selection criteria; he considers a weighted sum of squared error loss, an absolute error loss, and several score functions from Gneiting and Raftery (2007). One additional thought is that it would be interesting to see if an optimality result, like the result from the Appendix of BCS, exists for some (or any) of the alternatives. Without an optimality result, one might be hesitant to directly apply these loss functions to obtain locally selected predictions. Additionally, it would be interesting to gauge the performance of these alternative selection criteria in selecting the "true" model, as investigated by TS (with squared error loss).

\subsection{The predictor $\hat{k}(\cdot)$}

TS proposes using maps of $\hat{k}(\cdot)$ to determine whether or not the spatial process exhibits short-range or long-range dependence in certain regions. We agree, noting that this is directed more towards model building than the model selection studied in BCS. [See Box and Norman (1987), for a discussion on the differences between modeling building and model selection]. Model building is an area of spatial statistics that needs development and, echoing our comments in Section 1, the ideas in BCS could contribute to this literature.

In Sections 1,3.2, and 6 of BCS, we suggest using maps of $\hat{k}(\cdot)$ as an exploratory data analysis tool. In TS's Fig. 3, the disparate regions that were built into the simulation model are clear even though there is large variability in $\hat{k}(\cdot)$; this is consistent with the simulation results in Bradley et al. (2012). Thus, $\hat{k}(\cdot)$ appears to have utility as an exploratory data analysis tool, even in the setting where $\hat{k}(\cdot)$ has large variability.

Using the predictor $\hat{k}(\cdot)$ as a confirmatory tool is potentially problematic (as TS's simulation results suggest), since no optimality result exists for $\hat{k}(\cdot)$. Consequently, the variability of the estimated range parameter, the measurement error associated with a particular location, and the degree of discrepancy between the true range parameters in two disparate regions could all have an effect on the performance of $\hat{k}(\cdot)$. However, 
an optimality result does exist for the LSP; see the Appendix of BCS. Thus, to solve the model-building problem posed by TS, one might instead consider using a locally selected estimator (LSE) of the range parameter, which would require an extension of BCS's optimality result. If this result were available, maps of the LSE could determine regions with distinct range parameters in an optimal manner.

\section{Uncertainty quantification}

Two of the discussants (GE and CG) expressed concern that the LSP does not have uncertainty measures associated with. We agree that consistent uncertainty quantification is an important problem that needs to be developed for the LSP, and several suggestions for this future work were provided in Section 6 of BCS. The "bagging" idea that RM proposed has the potential to resolve this problem as well. That is, one could add a fifth step to the numbered list in Section 4.1 just above:

\section{Compute the sample variance across the $B$ LSPs generated in Step 3.}

The suggestions in Section 6 of BCS and RM's bagging idea require additional attention. For example, simulation studies analogous to those we conducted in BCS might be used to determine whether or not these ideas consistently capture the true variability of the LSP.

\section{Concluding remarks}

The literature on predictor selection is expansive for non-spatially referenced data; however, it needs development in the spatial data setting. The efforts of Chen et al. (2010), Zhu et al. (2010), Bradley et al. (2011), Chen and Huang (2011a,b), and Lai et al. (2012) have helped enormously in this area, but more research is needed. The appropriateness of a stochastic model depends largely on the underlying physical process and the data that are available. Consequently, simply arguing that an approach has limitations (e.g., see Stein, 2005, 2014) is not enough to address important model/predictor comparison problems. We propose that in an era of big spatial data, the empirical, algorithmic point of view used in BCS is better suited to solve these important problems, since we have the luxury of having enough data to separate them into training, validation, and testing data. Our perspective on spatial prediction is "big tent," and which spatial predictor is better depends largely on the spatial dataset!

\section{References}

Anselin L (1995) Local indicators of spatial association. Geogr Anal 27:93-115

Box G, Norman R (1987) Empirical model-building and response surfaces. Wiley, Oxford

Bradley JR, Cressie N, Shi T (2011) Selection of rank and basis functions in the spatial random effects model. In: Proceedings of the 2011 Joint Statistical Meetings. American Statistical Association, Alexandria, pp 3393-3406

Bradley JR, Cressie N, Shi T (2012) Local spatial-predictor selection. In: Proceedings of the 2012 Joint Statistical Meetings. American Statistical Association, Alexandria, pp 3098-3110 
Chen CS, Huang HC (2011a) Geostatistical model averaging based on conditional information criteria. Environ Ecol Stat 19:23-35

Chen C-S, Huang H-C (2011b) An improved Cp criterion for spline smoothing. J Stat Plan Inference $141: 445-452$

Chen Y-P, Huang H-C, Tu I-P (2010) A new approach for selecting the number of factors. Comput Stat Data Anal 54:2990-2998

Cressie N, Johannesson G (2006) Spatial prediction for massive data sets. In: Australian Academy of Science Elizabeth and Frederick White Conference. Australian Academy of Science, Canberra, pp 1-11

Cressie N, Johannesson G (2008) Fixed rank kriging for very large spatial data sets. J R Stat Soc Ser B 70:209-226

Cressie N, Wikle CK (2011) Statistics for spatio-temporal data. Wiley, Hoboken

Gneiting T, Raftery A (2007) Strictly proper scoring rules, prediction, and estimation. J Am Stat Assoc 102:359-378

Hastie T, Tibshirani R, Friedman J (2009) The elements of statistical learning: data mining, inference, and prediction. Springer, New York

Lai R, Huang H-C, Lee T (2012) Fixed and random effects selection in nonparametric additive mixed models. Electron J Stat 6:810-842

Stein M (2005) Space-time covariance functions. J Am Stat Assoc 100:310-321

Stein M (2014) Limitations on low rank approximations for covariance matrices of spatial data. Spatial Stat $8: 1-19$

Sun Y, Li B, Genton M (2012) Geostatistics for large datasets. In: Porcu E, Montero JM, Schlather M (eds) Space-time processes and challenges related to environmental problems. Springer, Berlin, pp 55-77

Zhu J, Huang H-C, Reyes P (2010) On selection of spatial linear models for lattice data. J R Stat Soc Ser B 72:389-402

Zimmerman D, Zimmerman M (1991) A comparison of spatial semivariogram estimators and corresponding ordinary kriging predictors. Technometrics 33:77-91 